\title{
Load Identification on an Arch Bridge Based on the Generalized Flexibility Matrix
}

\author{
Yu Wang ${ }^{1}$, Jianting Zhou ${ }^{1}$, Jingzhou Xin ${ }^{1} \&$ Linshan Cai ${ }^{1}$ \\ ${ }^{1}$ School of Civil Engineering \& Architecture, Chongqing Jiaotong University, Chongqing 400074, China \\ Correspondence: Yu Wang, School of Civil Engineering \& Architecture, Chongqing Jiaotong University, \\ Chongqing 400074, China. E-mail: trevor14@foxmail.com
}

\author{
Received: April 25, $2014 \quad$ Accepted: May 19, $2014 \quad$ Online Published: June 25, 2014 \\ doi:10.5539/mas.v8n4p127 URL: http://dx.doi.org/10.5539/mas.v8n4p127
}

\begin{abstract}
Health problems of the bridge structure are a hot issue in engineering research currently. Load identification together with damage identification is an important method to diagnose the health status of the bridge. In order to identify different loads on bridges, this paper takes a box arch bridge built with prestressed reinforced concrete in Chongqing as an example and establishes it's finite element model. The general flexibility matrix is obtained by calculating the response values of each control section separately caused by each unit characteristic load. The maximum bending moment of each control section is acquired by using the time history analysis of Wenchuan Seismic wave, and the equivalent loads are derived from the general flexibility matrix. Results indicate that this method can identify a wide range of basic load types and accurately identify the actual load of the bridge with high precision. Thus the actual operating conditions of bridge can be better reflected and the bridge's safety condition can be evaluated. Besides, the load evaluation and maintenance of the bridge can be based on this.
\end{abstract}

Keywords: generalized flexibility matrix, equivalent internal forces, finite element method, seismic wave, load identification

\section{Introduction}

With the development of national economy and increase of traffic volume and traffic density, damage accumulation of bridge structure will be caused as the bridge is repeatedly affected by traffic and natural causes. Thus, fatigue damage, even the fatigue rapture can be caused, threatening the bridge safety in use and reducing the service life of the bridge. For example, there is some phenomenon of destruction on the deck of many shortly used bridge, such as cracking, material unconsolidation and collapse, which cannot be explained by static theory; meanwhile, cracking emerges on many prestressed concrete bridges which have been designed with crack resistance, and the cracking may become worse due to the repeated action of traffic loads as well as the increase of operation time, consequently, corrosion of steel bar will be caused and the load capacity of the bridge will decrease. Therefore, if detailed research on bridge damage and destruction mechanism that are caused by various loads can be conducted and loads acting on the bridge can be identified and measured, it will be of great theoretical and applicational value in health monitoring, daily maintenance, safety assessment and traffic design of bridge. And it is also of important guiding significance in design, construction and maintenance of bridge structure.

Load identification of bridge structure is one of the most important issues in bridge design, and it is also an import aspect in inverse problem in structural dynamics. The research of identifying the load on bridge deck by means of bridge response has made some progress and the main identifying methods are: Frequency Domain Method, Frequency-Time Domain Method and methods based on neural network, etc. At present, the load identification methods at home and abroad mainly target at certainty load, which mainly include periodic dynamic load and impact load. Many researches show that the bridge response caused by dynamic load is stronger than that of static load, besides, dynamic load will cause more damage than static load does, usually 2-4 times (Cebon, 1987). The research of identification of random load as well as accidental load is still in progress and there is little achievement in this area.

Based on a prestressed reinforced concrete box arch bridge in Chongqing, this paper focuses on the time-history analysis of its finite element models under the effect of Wenchuan Earthquake Load and the actual response time-history of the bending moment of the key measure points of the bridge structure is achieved. According to 
the principal of equivalence of internal force, the traffic load can be regarded as concentrated load acting on each span of the bridge, thus, the most unfavorable load-bearing situation of the bridge when normally operates can be achieved. Data analysis together with testing results indicate that this method is of considerable accuracy and high practical applicability. Besides, it can be easily adopted at low cost, therefore, this method can be referred to when trying to obtain other bridges' load identification.

\section{The General Situation of the Bridge and the Establishing of Model}

The bridge in Chongqing is a two-way 4-lane prestressed reinforced box arch bridge, used as one of the main traffic thoroughfares by passengers and vehicles. The total length of this bridge is $133.20 \mathrm{~m}$ with a $75 \mathrm{~m}$ reinforced concrete box main span, and the approach bridges at both ends are made of $0.9 \mathrm{~m}$ prestressed hollow slab girders with a span of $20 \mathrm{~m}$; the width of the bridge is $10 \mathrm{~m}$, made up of $0.5 \mathrm{~m}$ ( sidewalk) $+9 \mathrm{~m}$ (vehicle road ) $+0.5 \mathrm{~m}$ (sidewalk); and a 1.36\% longitudinal slope is designed. The upper section of the main span is made of prestressed concrete hollow slab with the height of $22.5 \mathrm{~cm}$ and a span of $9 \times 9 \mathrm{~m}=81 \mathrm{~m}$. "U" gravity-type abutments are used at both ends. C50 concrete, C40 concrete and C30 concrete are used for main arch, girder and piers, uprights together with other ancillary facilities. 3D model of the bridge established by software SketchUp is shown in Figure 1.

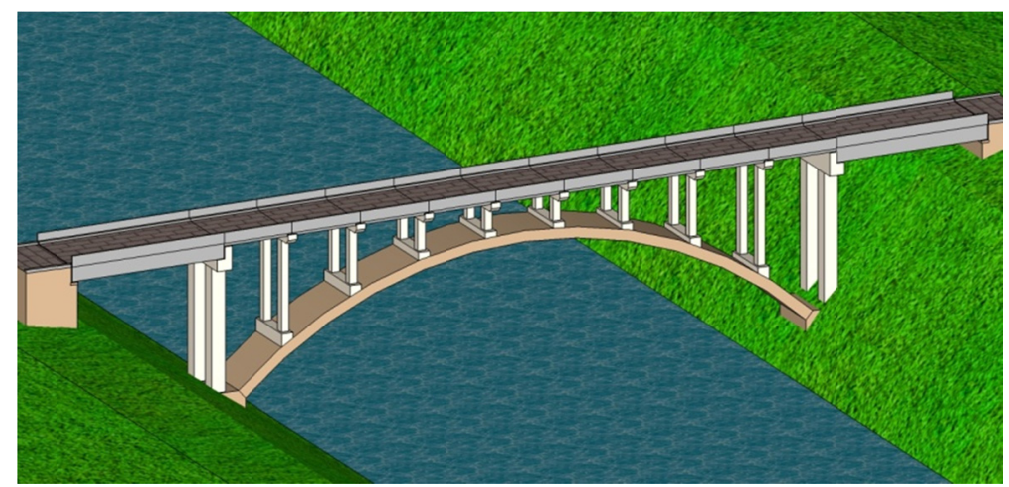

Figure 1. 3D model of the bridge

In order to continue the analysis, finite element model is established by using software SAP2000 V9.04. As the finite element model cannot indicate all the characteristics of the original bridge, proper simplification of the model has been conducted. Considering that the purpose of this paper is to identify the loads born by the bridge, which is conducted after the deformation caused by dead weight of the bridge and prestress, therefore, this paper does not take into consideration of the influence of the prestress. The whole structure includes 148 nodes and 126 elements. The finite element model of the bridge structure is shown in Figure 2.

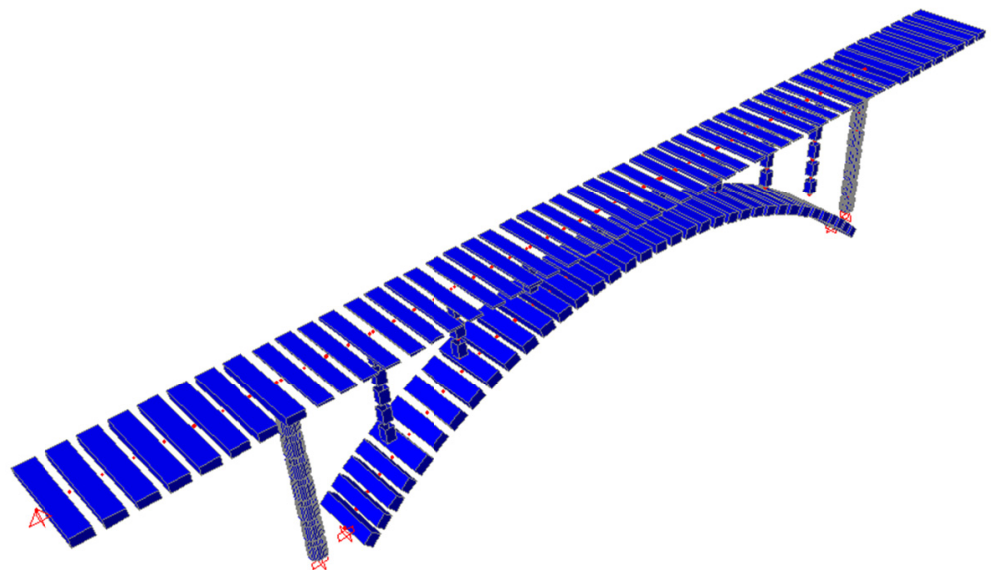

Figure 2. The finite element model of the bridge 


\section{The Establishing of Generalized Flexibility Matrix}

When traffic load or other kinds of load act on the bridge, relevant response will be caused at each structural position. Considering the the interaction of loads, the equivalent load that has the same effect as the actual load born by the bridge can be backwardly induced by establishing corresponding relationship between load and response as well as taking into consideration of the effect from each control point.

\subsection{The Basic Principal of Equivalence of Internal Force}

As to identify the actual load born by the bridge, the control cross section is set as $n$, the response value of a certain measure point $j(j=1, \cdots, n)$ caused by unite characteristic load $P_{i}$ is set as $\delta_{j i}$, thus, based on this situation, the equation of load control can be established:

$$
\left(\begin{array}{ccc}
\delta_{11} & \ldots & \delta_{1 n} \\
\vdots & \ddots & \vdots \\
\delta_{n 1} & \cdots & \delta_{n n}
\end{array}\right)\left(\begin{array}{c}
P_{1} \\
\vdots \\
P_{n}
\end{array}\right)=\left(\begin{array}{c}
\Delta_{1} \\
\vdots \\
\Delta_{n}
\end{array}\right)
$$

In this equation, $\left[\delta_{i j}\right]$ is the influence coefficient matrix, that is, the generalized flexibility matrix of structure; $\left[\Delta_{i}\right]$ is the matrix made up of actual response data at each control cross section measure point under the effect of actual load; $\left[P_{i}\right]$ stands for the number $i(i=1, \cdots, n)$ unite characteristic load, that is, the equivalent load obtained from this equation.

\subsection{The Establishing of Generalized Flexibility Matrix of Structure}

In order to get the generalized flexibility matrix, the response value of each control point under the effect of various unite characteristic load shall be calculated separately. There are various types of response values, such as absolute displacement, relative displacement, absolute intersection angle, relative intersection angle, stress, strain and bending moment. In this paper, the bending moment is taking as application examples.

The response value of each control cross section is gathered through the analysis of the action of each unite concentrated force, and thus the generalized flexibility matrix of the structure can be obtained. This paper will conduct its analysis based on the bending moment value of 41 control cross sections in the girder, thus, the $41 \times 41$ order generalized flexibility matrix can be obtained.

\subsection{The Load Equivalence}

To ensure the accuracy and correctness of the identification result, the control points have been chosen as many as possible in a reasonable range. As for this bridge, 7 control points are chosen at both approach bridges respectively and 3 control points are chosen from each span in the main bridge, therefore, there are totally $7 \times 2+3 \times 9=41$ control points. The concentrated force of these 41 control points has been used as the equivalent load form, also, the equivalent characteristic load, of the actual load. This is shown in Figure 3.

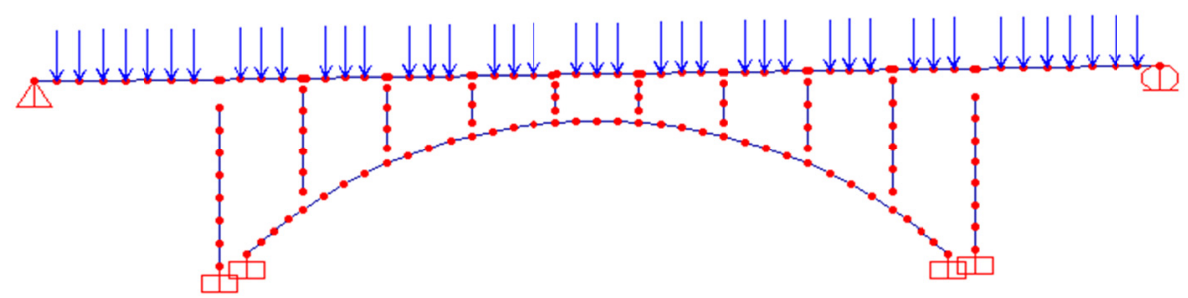

Figure 3. The equivalence of actual load

\subsection{The Solving of the Equivalent Characteristic Load}

After the obtaining of the response value of the bridge structure under the action of actual load, the numerical calculation software MATLAB can be adopted to program, make M documents and calculate. Therefore, various loads (including each concentrated load, distributed load, prestress, characteristic temperature variation, characteristic inertial force and bearing displacement) born by the bridge can be equaled to a linear combination of various characteristic loads, thus, the characteristic load coefficient of each structural control cross section, that is, the coefficient of linear combination of unite characteristic load, can be obtained. This method can be used to figure out the actual load acting on the bridge and imitate as well as simplify the stress state of the 
structure, better reflecting the actual operation situation of the bridge. The key to solve characteristic is to solve the control equation of the structure and there are three ways to do so by using software MATLAB, namely: LDL decomposition method, QR decomposition method and SVD decomposition method. The detailed way to use this and the applicable conditions are explained as follows:

(1)LDLT decomposition method: When the coefficient matrix of the equation is a symmetric matrix, the $L D L^{T}$ of matrix can be used to solve $A X=b$. This method can simplify the procedure and reduce the calculating amount;

(2)QR decomposition: To decompose matrix A into a orthogonal matrix $Q$ and an upper triangular matrix $R$, that is, $A=Q R$. There is no need to select a pivot element in this method and the calculation is stable but the calculation amount may be large;

(3) SVD decomposition: When $A$ is a $M \times N$ matrix instead of a square matrix, that is to say, the number of control cross section is not the same with that of the extraction points of actual responding data, Singular Value Decomposition can be adopted.

\section{Load Identification based on Data Collected from Wenchuan Earthquake}

To know and control the bearing capacity and operation condition of the bridge during the bridge operation period, especially under an accidental load, and to ensure the security, integrity, applicability and durability of the bridge structure, the most feasible method at present is to set up a health-monitoring system for bridge structures, observe the service security condition of bridge structures, and to evaluate the health condition of a bridge. The actual load of a bridge which is in operation equals to the value of characteristic load which can concluded from the measured value of the corresponding sensor in the health-monitoring system for bridge structures, and from generalized flexibility matrix set up according to the equivalence principle of internal forces. Mechanical parameters like deformation and stress of crucial positions of the bridge can be computed out, thus we can evaluate the security level of the bridge in accordance with those mechanical parameters.

\subsection{To Collect Actual Response Data from Time-History Analysis}

Since 1960s, time-history analysis is a seismic analytic method gradually developed, used for anti-seismic analysis of super-high buildings and for anti-seismic study of engineering projects. It has become one of the analytic methods for the majority of the countries in anti-seismic design specifications or regulations. In terms of basic motion equations of engineering projects, first input several time-history curves under seismic acceleration records or artificial acceleration corresponding to construction sites, and then work out, by integration, the whole changing process of internal forces and deformation of bridge structures over time under changing ground acceleration over time, and finally check the seismic resistant capacity and deformation of the cross section of structural elements.

To verity the reliability of this essay's method, this essay adopts data of the Wenchuan earthquake wave. There being no health-monitoring system of bridge structures for this bridge, and no actual monitoring data, this essay defines this bridge with SAP2000 V9.04 time history function, make time history analysis with Wenchuan earthquake wave data. After analogous simulation of the real situation, this essay takes the bending moment of each cross sections in the analytic results of their internal forces as the actual response of bridge structures. The time history function of Wenchuan earthquake waves is demonstrated in Figure 4.

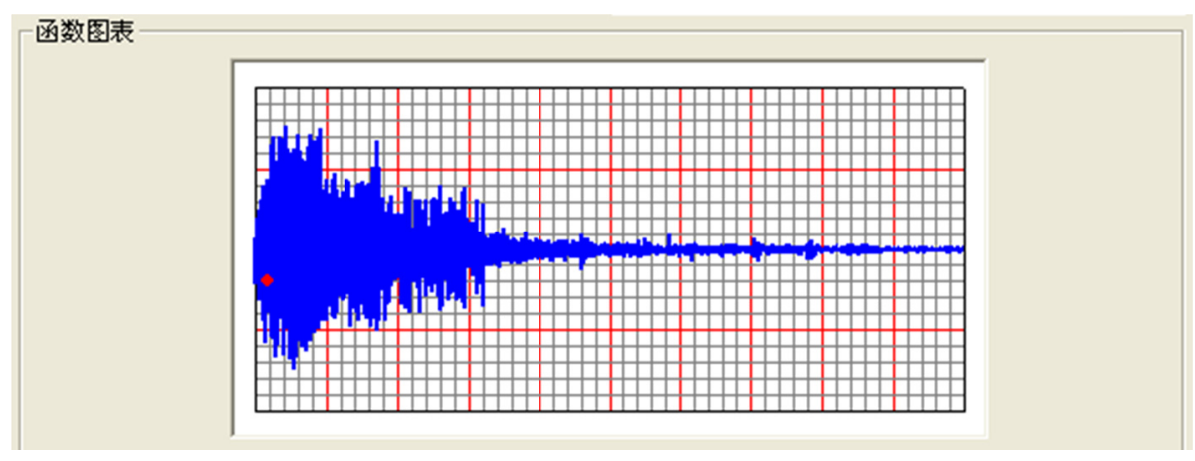

Figure 4. The time history function of Wenchuan earthquake waves 


\subsection{Identification of the Maximum Value of Seismic Load}

After time history analysis, choose the maximum moments of 41 control sections as the actual response of bridge structures, and program by MATLAB and then establish $M$ document for computation. After inputing the generalized flexibility matrix obtained from the former session and the extracted actual response into programs, coefficient of load linear combination with unit features. Equivalent load of the actual load of bridge structures can be figured out after simultaneously exerting forces on the 41 control sections that are the product of the 41 unit concentrated force and their corresponding coefficient of feature load as shown in the Figure5.

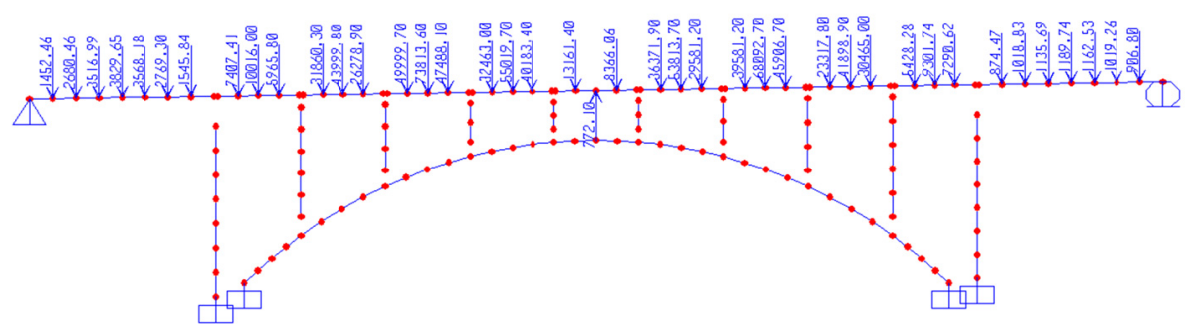

Figure 5. Equivalent load made of feature load

\subsection{Analysis of the Conformity Degree of Load Recognition Results}

Use the finite element software SAP2000 to analyze the equivalent load as shown in Figure5, and extract the result of internal forces, and then the values of bending moment of each control section can be obtained under the equivalent load. And then input values of bending moment of main control points respectively under equivalent load and actual load into table1. To better illustrate disparities of bridge structures caused by two different loads, a bending moment line chart of control sections under the two conditions can be drawn according to table1, as shown in Figure 6.

Table 1. Values of bending moment of main control points under equivalent load and actual load (unit: $\mathrm{kN} \cdot \mathrm{m}$ )

\begin{tabular}{lccccccccc}
\hline Node number & 804 & 810 & 818 & 822 & 826 & 830 & 834 & 842 & 848 \\
\hline $\begin{array}{l}\text { Bending moment } \\
\text { under equivalent }\end{array}$ & 61968.33 & 35914.64 & 263800 & 196900 & 21480.83 & 187200 & 238700 & 33731.44 & 22174.00 \\
$\begin{array}{l}\text { load } \\
\text { Bending moment } \\
\text { under actual load }\end{array}$ & 61828.31 & 35910.73 & 263500 & 196400 & 21480.77 & 186600 & 238300 & 33671.73 & 21451.11 \\
\begin{tabular}{l} 
Relative error/\% \\
\hline
\end{tabular} & 0.226466 & 0.010887 & 0.113852 & 0.254582 & 0.000270 & 0.321543 & 0.167855 & 0.177349 & 3.369924 \\
\hline
\end{tabular}

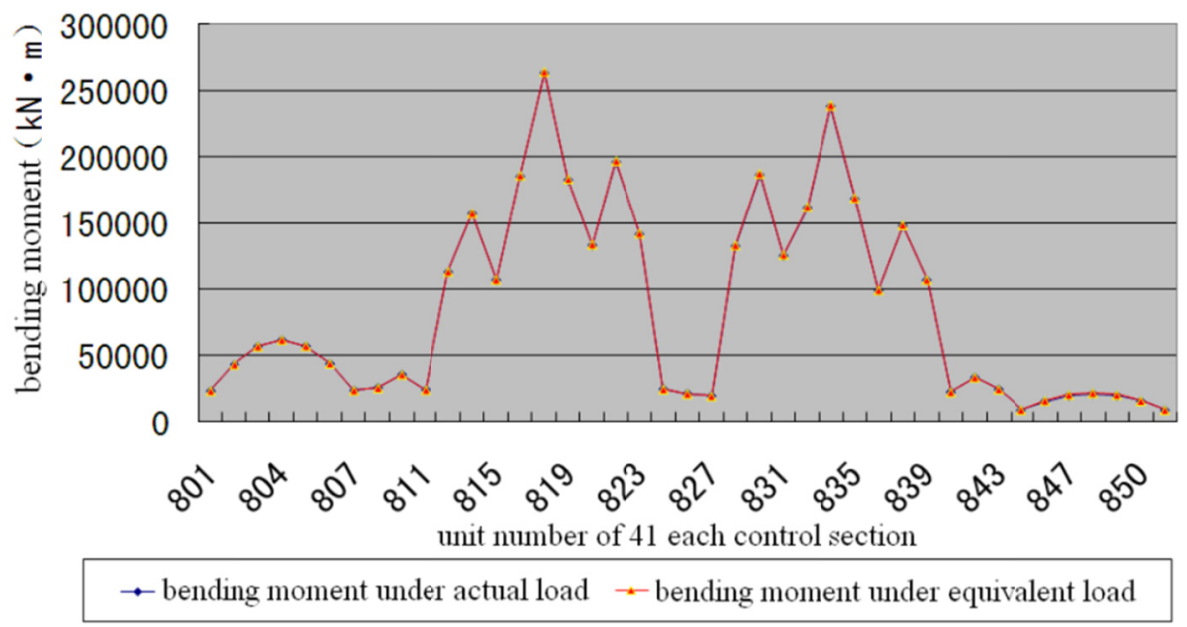

Figure 6. Line chart of bending moment under equivalent load and actual load 
From Figure 6, it can be seen that the values of bending moment under equivalent load and actual load are highly conformable. The results indicate that the changing rules of bending moment computed out of identified equivalent feature load and of bending moment of cross sections under actual earthquake load are identical, and the absolute error as well as the relative error is slight, satisfying the accuracy requirement of bridge security evaluation in engineering projects, which fully demonstrates that the idea of substituting actual load with equivalent load is both feasible and pragmatic.

\section{Conclusion}

This essay, on the background of stress reinforced concrete box arched bridge, set up finite element models, and verifies the effectiveness and pragmatism of substituting actual load with equivalent feature load according to the equivalence principle of internal forces by identifying the actual load during the Wenchuan earthquake by means of generalized flexibility matrix of itself. And this essay draws the following conclusions:

(1) The errors of the results inducted from the equivalence principle of internal forces for the Wenchuan earthquake load are all less than 5\%, most of which are less than $1 \%$, which fully demonstrates that it is feasible to substitute actual load with equivalent load. The method stated in this essay can be used in various types of basic loads, and various types of information can be measured out. The actual load of the bridge can also be figured out fairly accurately which can reflect the actual operation condition of the bridge so as to evaluate the security condition of the bridge.

(2) As for the bridges which already have structural health-monitoring systems set up, they can take the actual load during the operation as the equivalent feature load which is fast, pragmatic, and accurate in an acceptable scope of engineering projects.

(3) The equivalence principle of internal forces based load identification method of this essay is able to get the equivalent feature response under actual load. When the response is highly representative, the accuracy of load evaluation can be increased pronouncedly, which provides evidence for the maintenance and management of the bridge's regular operation.

\section{References}

Airong Chen, Rujin Ma., \& Yanmei, Xu. (2013). Vehicle Loading Identification and Its Characteristics of Sutong Bridge in Operation. Journal of Chongqing Jiaotong University(Natural Science). Retrieved from http://222.198.130.63/kcms/detail/detail.aspx?QueryID=0\&CurRec=1\&DbCode=CJFD\&dbname=CJFDLA ST2013\&filename $=$ CQJT2013S1002\&uid=aTF6cnduaEZ6Y1R6MFVXcWNGek5zOVIYVFhrWGhiandQ Q1JKQXpWbXFtSytha3VT

Deng, L., \& Cai, C. S. (2010). Identification of dynamic vehicular axle loads: theory and simulations. Journal of Vibration and Control, 16(14), 2167-2194. http://dx.doi.org/10.1177/1077546309351221

Longlong, Li., \& Lingyi, Lu. (2012). Identification of the Tall Structure Wind Load Based on Pseudo-excitation Method. Construction \& Design for Project. Retrieved from $\mathrm{http} / / / 222.198 .130 .63 / \mathrm{kcms} /$ detail/detail.aspx?QueryID=1\&CurRec=1\&DbCode=CJFD\&dbname=CJFDLA ST2013\&filename=ZDCJ201303029\&uid=aTF6cnduaEZ6Y1R6MFVXcWNGek5zOV1YVFhrWGhiandQ Q1JKQXpWbXFtSytha3VT

Zhong Xian, Li., \& Feng Chen. (2006). IDENTIFICATION AND PARAMETRIC ANALYSIS OF MOVING LOADS ON SIMPLY SUPPORTED AND MULTI-SPAN CONTINUOUS BRIDGES. Engineering Mechanics, 23(12), $\quad$ 91-99. $\quad$ Retrieved from http://222.198.130.63/kcms/detail/detail.aspx?QueryID=6\&CurRec=1\&DbCode=CJFD\&dbname=CJFD06 08\&filename $=$ GCLX200612016\&uid=aTF6cnduaEZ6Y1R6MFVXcWNGek5zOV1YVFhrWGhiandQQ1JK QXpWbXFtSytha3VT

Ou, J. P., \& Li, H. (2010). Structural health monitoring in mainland China: Review and future trend. Structural Health Monitoring-An International Journal, 9(3), 219-231. http://dx.doi.org/10.1177/1475921710365269

Yuantian, Qin, Guoping, Chen, Yuling, \& Zhang fang. (2008). Moving Load Identification Using Finite Element Method for Complex Structure[J]. Journal of Nanjing University of Aeronautics \& Astronautics, 40(2), 174-179. Retrieved from http://222.198.130.63/kcms/detail/detail.aspx?QueryID $=4 \&$ CurRec $=1 \&$ DbCode $=$ CJFD $\&$ dbname $=$ CJFD0608 \& filename $=$ NJHK200802009\&uid $=$ aTF6 cnduaEZ6Y 1R6MFVXcWNGek5zOVlYVFhrWGhiandQQ1JKQXpWbXFtSytha3VT

Lei, Wang, Jilin, Hou, \& Jin-ping Ou. (2012). Moving force identification based on load shape function for a long-span bridge structure. Chinese Journal of Computational Mechanics, 2, 153-158. Retrieved from 
http://222.198.130.63 $/ \mathrm{kcms} /$ detail $/$ detail.aspx $?$ QueryID $=0 \&$ CurRec $=1 \& D b C o d e=C J F D \&$ dbname $=$ CJFD 111 2\&filename=JSJG201202000\&uid=aVpZV1Q5QzBibjRIS0tpLzdSR2xHbUJlcjRmYkJYUm1MWExQVllJ $\mathrm{MkVpQXFpU3Np}$

Ning-bo, Wang, Weixin, Ren, \& Miao, Li. (2013). Moving load identification of a bridge based on influence line. Journal of Vibration and Shock. Retrieved from http://222.198.130.63 $/ \mathrm{kcms} /$ detail $/$ detail.aspx?QueryID=1\&CurRec $=1 \& D b C o d e=C J F D \&$ dbname=CJFDLA ST2013\&filename=ZDCJ201303029\&uid=aTF6cnduaEZ6Y1R6MFVXcWNGek5zOVIYVFhrWGhiandQ QIJKQXpWbXFtSytha3VT

Dongsheng, Xu., \& Jinping, Ou. (2009). Identifying of Vehicle Load Based on Information Obtained from Health Monitoring System for Long-span Bridge. Southeast University: Proceedings of the 7th National Civil Engineering Forum for graduate Students (NCFEGS 2009).

Qingxia, Zhang, Zhongdong, Duan \& Lukasz Jankowski. (2012). The study on simultaneous identification of structural damages and loads. Engineering Mechanics. Retrieved from http://222.198.130.63 $/ \mathrm{kcms} /$ detail $/$ detail.aspx?QueryID=3\&CurRec=1 $\&$ DbCode=CJFD\&dbname $=$ CJFDLA ST2013\&filename $=$ GCLX201212046\&uid=aTF6cnduaEZ6YlR6MFVXcWNGek5zOVlYVFhrWGhiandQ QIJKQXpWbXFtSytha3VT

\begin{abstract}
Authors
Yu Wang (1990-). Male, from Daming County, Hebei Province, postgraduate in Chongqing Jiaotong University. His major research fields are the detection, evaluation of bridge, and also mechanical analysis.

Jianting Zhou (1972-). Male, from Jinhua, Zhejiang Province, professor of Chongqing Jiaotong University, and also a doctor and a doctoral supervisor of Chongqing jiaotong University.

Jianting Zhou, with a doctorate degree, is a post-doctoral, professor as well as doctoral supervisor. Receiving special government allowance from the State Council, he has achieved many honors including "New Century Excellent Talents in University of Ministry of Education of China ", "the 4th Ten Outstanding Young Persons of Chongqing", "the 1st Outstanding Mid-Aged Experts of Chongqing", "the 1st Preeminent Youth Fund of Chongqing", "the 2nd Scientific and Technological Award of Highway Youths of China" etc. Professor Zhou's research fields are structural security observation, evaluation and reinforcement. He has also hosted and led as much as 20 projects of national and provincial and departmental levels, including national key scientific and technological key projects, and projects of National Science Foundation of China. He has also led 25 horizontal projects. Professor Zhou has achieved 13 scientific and technological innovations, published 138 academic treatises including 32 in SCI, EI, ISTP, 2 academic books, and honored 7 awards of science and technology at the provincial and departmental levels(1 first prize, 3 second prize). He has applied for 25 patents, 9 of which are already approved of (5 inventive patens and 4 new pragmatic patents), the rest 16 of which are already published.
\end{abstract}

\title{
Copyrights
}

Copyright for this article is retained by the author(s), with first publication rights granted to the journal.

This is an open-access article distributed under the terms and conditions of the Creative Commons Attribution license (http://creativecommons.org/licenses/by/3.0/). 